\title{
HYBRID VIGOR IN SUNFLOWER (Helianthus annuus L.)
}

\author{
Yalcin Kaya $^{*}$
}

Trakya Agricultural Research Institute, P.O. Box 16, 22100 Edirne, Turkey

Received: December 12, 2004 Accepted: November 22, 2005

\author{
SUMMARY
}

Increasing seed and oil yields is the top priority of most sunflower breeding programs. Getting benefit from use of heterosis is the main purpose in sunflower hybrid breeding. The objectives of this study were to determine performance of sunflower varieties and to measure the vigor of sunflower hybrids. In 2000 and 2001, sunflower hybrids were evaluated for four important yield components, yield performance, standard and regular heterosis, and heterobeltiosis, in Edirne - Turkey conditions. Based on observations in this research, seed yield of hybrids was changed between 37 and $245 \mathrm{~kg} \mathrm{ha}^{-1}$, oil yield between 17.6 and $118.8 \mathrm{~kg} \mathrm{ha}^{-1}$, oil content between 38.0 and $50.8 \%$ and hull rate between 19.2 and $27.1 \%$. The highest heterosis $(288.3 \%)$ and heterobeltiosis (98\%) were found for oil yield. The highest standard heterosis $(21.2 \%)$ was computed for seed yield. The lowest heterosis $(-19.3 \%)$ and heterobeltiosis $(-22.4 \%)$ were observed for hull rate. The lowest standard heterosis $(-22.0 \%)$ was measured for oil yield. Regarding the studied traits, most favorable performances were shown by the $11^{\text {th }}$ cross $(2453-\mathrm{A} \times 2644-\mathrm{R})$. Among inbred lines, the female 2453-A and the male 2644-R exhibited higher hybrid vigor than the others.

Key words: sunflower, hybrid breeding, heterosis, heterobeltiosis, hybrid vigor

\section{INTRODUCTION}

Sunflower (Helianthus annuus L.) is grown worldwide, mostly as a source of vegetable oil and proteins. The main objectives of sunflower breeding programs are the development of productive $F_{1}$ hybrids with high seed and oil yield. Sunflower oil yield is determined as the product of seed yield per unit area and the oil percentage in grains. Therefore, consideration of both components is important when breeding for high oil yield (Fick and Miller, 1997).

* Corresponding author, Phone: 90284235 8182, Fax: 902842358210 , e-mail: yalcinkaya@ttae.gov.tr 
Seed oil and hull content are the main characteristics determining oil yield in sunflower. Oil content is a quantitatively inherited trait and it varies considerably depending on cultivar and also environmental effects (Miller, 1987). There is a negative correlation between hull and oil contents of seed. Miller and Fick (1997) indicated that most of the increase in seed oil from past sunflower breeding and selection resulted from decreases in hull percentage of seed. Pathak et al. (1983) detected negative increases in oil content of sunflower hybrids and they concluded that the reasons for that were possibly overdominance effects of the genes conferring low oil content and the same frequencies of these genes in hybrids and their parents.

Heterosis, defined as the unusual growth and yield of heterozygous hybrids from two less vigorous homozygous parents, has been explored during last 70 years. Heterosis can be considered as one of the most important contributions of the genetics to agriculture, with large consequences on agricultural yields. Farmers generally prefer hybrids developed by utilizing heterosis to cultivars and grow them on large areas. Sunflower hybrids predominate the sunflower productions in Turkey and in the world (Kaya, 2004).

Although the hybrid vigor can be generally defined as the superiority of individuals from the $\mathrm{F}_{1}$ generation in relation to their parents, it can also be considered as the superiority of new hybrids over currently grown commercial hybrids. This phenomenon has been analyzed for many different traits of sunflower. Researchers have observed considerable increases of heterosis for seed and oil yield and oil content in sunflower. Reddy et al. (1985) measured heterosis rates higher than $100 \%$ and heterobeltiosis rates higher than $10 \%$ in sunflower hybrids. Singh et al. (1984) observed heterosis rates from 47 to $206 \%$ in seed yield. Guo-Zhan and Chun-Fang (1985) reported the heterosis rates of $169 \%$ for seed yield, $2.3 \%$ for hull rate and $14.6 \%$ for oil content of sunflower. Giriraj and Virupakshappa (1992) and Virupakshappa et al. (1997) observed that the hybrid vigor for sunflower seed yield remained at the same level in different environments. Zhao-Cheng et al. (1988) also observed high heterosis in seed yield, oil and hull content and concluded that the parents having larger head diameter and seed volume had higher yield potential. This study was conducted to determine sunflower parents and hybrids with highest potential and hybrid vigor for seed and oil yields and hull and oil contents.

\section{MATERIAL AND METHODS}

Cultivated sunflower is primarily grown from single-cross hybrid seed. Therefore, the study was started with $25 \mathrm{~F}_{1}$ single-cross hybrids produced by crossing 5 male restorer lines to 5 cytoplasmic male sterile ( $\mathrm{cms}$ ) lines in the breeding nursery of Trakya Agricultural Research Institute in Edirne in 1999. The female lines (BAH4-A, BAH8-A, HA-89-A, 0704-A and 2453-A) and the male lines (2644-R, 2284-R, 2280-R, 25711-R and R-01001) come from National Sunflower Research 
Project. These parent lines were planted separately at Edirne experiment field in 2000 and 2001. The experiments were conducted in $6 \mathrm{~m}$ long, three-row plots with $70 \times 35 \mathrm{~cm}$ plant density. The middle row was harvested. The plot size at harvest was $3.78 \mathrm{~m}^{2}$.

The experimental design was randomized complete block design and it was used for both, parents (5 maintainer (B) females and restorers) and hybrids, in three replications. Seed and oil yield $\left(\mathrm{kg} \mathrm{ha}^{-1}\right)$, oil content (\%) and hull ratio (\%) were measured in this experiment. The magnitude of relative heterosis, heterobeltiosis and standard heterosis were estimated to facilitate the exploitation of hybrid vigor through heterosis breeding. Heterosis was computed using three measurements: mid-parent heterosis (calculated as the percentage of deviation from the mid-parent value), better-parent heterosis or heterobeltiosis (calculated as the percentage of deviation from the better-parent value) and standard heterosis (calculated as the percentage of deviation from the average of commercial hybrids). The \% heterosis was calculated from the formula:

$\%$ heterosis $(\mathrm{HTS})=100 \times\left(\mathrm{F}_{1}-\mathrm{MP}\right) / \mathrm{MP}$,

where $\mathrm{F}_{1}$ is the hybrid progeny and MP is the "mid-parent" or the average of the parents $(\mathrm{P} 1+\mathrm{P} 2) / 2$. The \% heterobeltiosis was calculated from the formula:

$\%$ heterobeltiosis $(\mathrm{HTB})=100 \times\left(\mathrm{F}_{1}-\mathrm{HP}\right) / \mathrm{HP}$,

where heterobeltiosis is the parent with highest phenotypic expression.

The standard heterosis was calculated from the formula:

$\%$ standard heterosis $(\mathrm{SHS})=100 \times\left(\mathrm{F}_{1}-\mathrm{SA}\right) / \mathrm{SA}$,

where SA is the average of commercial standard hybrids $(\mathrm{H} 1+\mathrm{H} 2) / 2$.

Commercial standard checks were TR-6149 ( $13^{\text {th }}$ cross, $\left.2453-A \times 2280-R\right)$ and TR-3080 $\left(15^{\text {th }}\right.$ cross, $2453-A \times$ R-01001) as registered sunflower hybrids in Turkey.

\section{RESULTS AND DISCUSSION}

Seed yield is an exceedingly complex quantitative trait in sunflower, whose control involves a series of genes, because practically all traits have some influence, to a large or small measure, on the seed yield. However, heterosis occurred practically for all traits with different magnitudes. The highest positive heterosis observed for seed yield was explained by the sum of favorable values of heterosis for the different traits correlated with seed yield.

Maximum hybrid vigor was measured in the $11^{\text {th }}$ cross $(25453-\mathrm{A} \times 2644-\mathrm{R})$ as heterosis $274.6 \%$ and heterobeltiosis $257.2 \%$ in the 2000 growing season. The lowest heterosis for seed yield was $23.0 \%$, observed in the $10^{\text {th }}$ cross, BAH- $8 \times \mathrm{R}$ 01001 , and the lowest heterobeltiosis was observed in the $14^{\text {th }}$ cross, $2453-\mathrm{A} \times$ 25711-R (Table 1). The evaluation of hybrids for heterosis breeding based on standard heterosis led to the identification of different sets of hybrids. Therefore, the evaluation of hybrids based on all three criteria would be more meaningful. Viewed from this angle, the following hybrids were considered as best: the $20^{\text {th }}$ cross (0704-A $\times$ R-1001), the $25^{\text {th }}$ cross (HA89-A $\times$ R-1001) and $11^{\text {th }}$ cross $(2453-$ 
A $\times$ 2644-R). Relative heterosis for seed yield ranged between $23.0 \%$ and $274.6 \%$ coupled with significant heterobeltiosis from -5.7 to $257.2 \%$. The standard heterosis for seed yield ranged from -20.9 to $21.2 \%$.

Table 1: Seed yields of the crosses tested in 2000 and 2001

\begin{tabular}{|c|c|c|c|c|c|c|c|c|c|c|c|c|c|c|}
\hline \multirow{2}{*}{\multicolumn{3}{|c|}{ CROSS $(A \times R)$}} & \multicolumn{6}{|c|}{2000} & \multicolumn{6}{|c|}{2001} \\
\hline & & & \multirow{2}{*}{$\begin{array}{c}\begin{array}{c}\text { HBT } \\
\mathrm{kg} \mathrm{ha}^{-1}\end{array} \\
2069\end{array}$} & \multirow{2}{*}{$\begin{array}{c}\begin{array}{c}\text { SHS } \\
\%\end{array} \\
0.7\end{array}$} & \multirow{2}{*}{$\begin{array}{r}\begin{array}{c}\text { Fem } \\
\mathrm{kg} \mathrm{ha}^{-1}\end{array} \\
1713\end{array}$} & \multirow{2}{*}{$\begin{array}{c}\begin{array}{c}\text { Male } \\
\mathrm{kg} \mathrm{ha}^{-1}\end{array} \\
608\end{array}$} & \multirow{2}{*}{$\begin{array}{c}\begin{array}{c}\mathrm{HTS} \\
\%\end{array} \\
78.3\end{array}$} & \multirow{2}{*}{$\begin{array}{c}\begin{array}{c}\text { HTB } \\
\%\end{array} \\
20.8\end{array}$} & \multirow{2}{*}{$\begin{array}{c}\begin{array}{c}\mathrm{HBT} \\
\mathrm{kg} \mathrm{ha}^{-1}\end{array} \\
1381\end{array}$} & \multirow{2}{*}{$\begin{array}{c}\begin{array}{c}\text { SHS } \\
\%\end{array} \\
5.3\end{array}$} & \multirow{2}{*}{$\begin{array}{r}\begin{array}{c}\text { Fem } \\
\mathrm{kg} \mathrm{ha}^{-1}\end{array} \\
904\end{array}$} & \multirow{2}{*}{$\begin{array}{l}\begin{array}{l}\text { Male } \\
\mathrm{kg} \mathrm{ha}^{-1}\end{array} \\
594\end{array}$} & \multirow{2}{*}{$\begin{array}{c}\begin{array}{c}\text { HTS } \\
\%\end{array} \\
84.5\end{array}$} & \multirow{2}{*}{$\begin{array}{c}\mathrm{HTB} \\
\% \\
52.9\end{array}$} \\
\hline 1 & BAH4-A & $\times 2644-R$ & & & & & & & & & & & & \\
\hline 2 & " & $\times 2284-R$ & 2247 & 9.4 & 1713 & 851 & 75.3 & 31.1 & 1167 & -11.1 & 904 & 556 & 59.9 & 29.2 \\
\hline 3 & $"$ & $\times 2280-R$ & 2130 & 3.7 & 1713 & 602 & 84.0 & 24.4 & 1290 & -1.7 & 904 & 545 & 78.1 & 42.8 \\
\hline 4 & “ & $\times 25711 R$ & 2063 & 0.4 & 1713 & 823 & 62.7 & 20.4 & 1244 & -5.2 & 904 & 604 & 65.1 & 37.6 \\
\hline 5 & " & $\times \mathrm{R}-1001$ & 2276 & 10.8 & 1713 & 1108 & 61.3 & 32.8 & 1108 & -15.5 & 904 & 710 & 37.4 & 22.6 \\
\hline 6 & BAH8-A & $\times 2644-R$ & 1918 & -6.6 & 1560 & 608 & 76.9 & 22.9 & 1179 & -10.1 & 1223 & 594 & 29.9 & -3.6 \\
\hline 7 & " & $\times 2284-R$ & 2179 & 6.1 & 1560 & 851 & 80.8 & 39.7 & 1336 & 1.8 & 1223 & 556 & 50.2 & 9.2 \\
\hline 8 & $"$ & $\times 2280-R$ & 2093 & 1.9 & 1560 & 602 & 93.6 & 34.2 & 1230 & -6.2 & 1223 & 545 & 39.1 & 0.6 \\
\hline$y$ & “ & $\times 25711 R$ & 1981 & -3.6 & 1560 & 823 & 66.3 & 27.0 & 1306 & -0.5 & 1223 & 604 & 43.0 & 6.8 \\
\hline 10 & $"$ & $\times \mathrm{R}-1001$ & 2085 & 1.5 & 1560 & 1108 & 56.3 & 33.7 & 1189 & -9.4 & 1223 & 710 & 23.0 & -2.8 \\
\hline 11 & $2453-A$ & $\times 2644-R$ & 2393 & 16.5 & 670 & 608 & 274.6 & 257.2 & 1506 & 14.8 & 1240 & 594 & 64.3 & 21.4 \\
\hline 12 & " & $\times 2284-R$ & 2303 & 12.1 & 670 & 851 & 202.9 & 170.7 & 1354 & 3.2 & 1240 & 556 & 50.8 & 9.2 \\
\hline 13 & $"$ & $\times 2280-R$ & 1841 & -10.4 & 670 & 602 & 189.4 & 174.8 & 1417 & 8.0 & 1240 & 545 & 58.7 & 14.3 \\
\hline 14 & $"$ & $\times 25711 R$ & 1918 & -6.6 & 670 & 823 & 156.9 & 133.0 & 1169 & -10.9 & 1240 & 604 & 26.8 & -5.7 \\
\hline 15 & “ & $\times \mathrm{R}-1001$ & 2266 & 10.3 & 670 & 1108 & 154.8 & 104.4 & 1207 & -8.0 & 1240 & 710 & 23.8 & -2.7 \\
\hline 16- & 0704-A & $\times 2644-R$ & 2047 & -0.3 & 1121 & 608 & 136.8 & 82.6 & 1446 & 10.2 & 1191 & 594 & 62.1 & 21.4 \\
\hline 17 & $"$ & $\times 2284-R$ & 2373 & 15.5 & 1121 & 851 & 140.7 & 111.7 & 1315 & 0.2 & 1191 & 556 & 50.5 & 10.4 \\
\hline 18 & " & $\times 2280-R$ & 2121 & 3.3 & 1121 & 602 & 146.2 & 89.3 & 1350 & 2.9 & 1191 & 545 & 55.5 & 13.3 \\
\hline 19 & " & $\times 25711 R$ & 2188 & 6.5 & 1121 & 823 & 125.1 & 95.2 & 1301 & -0.8 & 1191 & 604 & 45.0 & 9.2 \\
\hline 20 & $"$ & $\times \mathrm{R}-1001$ & 2490 & 21.2 & 1121 & 1108 & 123.4 & 122.2 & 1322 & 0.8 & 1191 & 710 & 39.1 & 11.0 \\
\hline 21 & HA89A & $\times 2644-R$ & 2196 & 6.9 & 1200 & 608 & 142.9 & 83.0 & 1213 & -7.5 & 1075 & 594 & 45.4 & 12.8 \\
\hline 22 & “ & $\times 2284-R$ & 2010 & -2.1 & 1200 & 851 & 96.0 & 67.5 & 1341 & 2.2 & 1075 & 556 & 64.4 & 24.8 \\
\hline 23 & " & $\times 2280-R$ & 1842 & -10.3 & 1200 & 602 & 104.4 & 53.5 & 1086 & -17.2 & 1075 & 545 & 34.0 & 1.0 \\
\hline 24 & $"$ & $\times 25711 R$ & 1733 & -15.6 & 1200 & 823 & 71.3 & 44.4 & 1038 & -20.9 & 1075 & 604 & 23.7 & -3.4 \\
\hline 25 & $"$ & $\times \mathrm{R}-1001$ & 2450 & 19.3 & 1200 & 1108 & 112.3 & 104.1 & 1270 & -3.2 & 1075 & 710 & 42.4 & 18.2 \\
\hline
\end{tabular}

Based on the average values of the two years, 2453-A, 0704-A, HA-89, BAH-4-A and BAH-8-A were best female parents regarding the regular heterosis for seed yield. The lines 2644-R, 2280-R, 2284-R, 25711-R and R-01001 were best restorers. Regarding heterobeltiosis, 2453-A, 0704-A, HA-89, BAH-8-A and BAH-4-A were best female parents and 2644-R, 2284-R, 2280-R, R-01001 and 25711-R were best male parents.

The values of heterosis in sunflower are highly variable for the different agronomic traits, especially for seed yield. Positive heterosis for this trait has been men- 
tioned in several papers. High heterosis values for sunflower seed yield, similar to those obtained in this research, were reported by Singh (1984), Guo-Zhan and Chung-Fang (1985), Reddy et al. (1985), Zao Cheng et al. (1988), and Giriraj and Virupakshappa (1992).

Heterosis for oil yield was as high as this for seed yield, especially in 2000. The values of heterosis, heterobeltiosis and standard heterosis changed between 15.3 and $288.3 \%,-13.3$ and $278.9 \%$ and $-22.0 \%$ and $21.0 \%$, respectively (Table 2). Maximum values of $288.3 \%$ for heterosis and $278.9 \%$ for heterobeltiosis were recorded for oil yield in 2453-A $\times 2644-\mathrm{R}$ in 2000 , followed by $2453-\mathrm{A} \times 2284-\mathrm{R}$ and $2453-\mathrm{A}$ $\times 2280-\mathrm{R}$. The maximum standard heterosis $(288.3 \%)$ was registered in the cross HA89-A $\times$ R-1001.

Table 2: Oil yields of the crosses in 2000 and 2001

\begin{tabular}{|c|c|c|c|c|c|c|c|c|c|c|c|c|c|c|}
\hline \multirow{2}{*}{\multicolumn{3}{|c|}{ CROSS $(A \times R)$}} & \multicolumn{6}{|c|}{2000} & \multicolumn{6}{|c|}{2001} \\
\hline & & & $\begin{array}{l}\mathrm{HBT} \\
\mathrm{kg} \mathrm{ha}^{-1}\end{array}$ & $\begin{array}{c}\text { SHS } \\
\%\end{array}$ & $\begin{array}{c}\text { Fem } \\
\mathrm{kg} \mathrm{ha}^{-1}\end{array}$ & $\begin{array}{c}\text { Male } \\
\mathrm{kg} \mathrm{ha}^{-1}\end{array}$ & $\begin{array}{l}\text { HTS } \\
(\%)\end{array}$ & $\begin{array}{l}\text { HTB } \\
(\%)\end{array}$ & $\begin{array}{l}\mathrm{HBT} \\
\mathrm{kg} \mathrm{ha}^{-1}\end{array}$ & $\begin{array}{c}\text { SHS } \\
\%\end{array}$ & $\begin{array}{c}\text { Fem } \\
\mathrm{kg} \mathrm{ha}^{-1}\end{array}$ & $\begin{array}{c}\text { Male } \\
\mathrm{kg} \mathrm{ha}^{-1}\end{array}$ & $\begin{array}{l}\text { HTS } \\
\text { (\%) }\end{array}$ & $\begin{array}{l}\text { HTB } \\
(\%)\end{array}$ \\
\hline 1 & BAH4-A & $\times 2644-R$ & 1011 & 2.1 & 848 & 299 & 76.2 & 19.2 & 617 & 10.7 & 352 & 271 & 98.0 & 75.2 \\
\hline 2 & " & $\times 2284-R$ & 1072 & 8.2 & 848 & 427 & 68.2 & 26.5 & 498 & -10.7 & 352 & 267 & 60.9 & 41.4 \\
\hline 3 & “ & $\times 2280-R$ & 1020 & 3.0 & 848 & 317 & 75.1 & 20.3 & 561 & 0.6 & 352 & 239 & 89.7 & 59.2 \\
\hline 4 & “ & $\times 25711 R$ & 935 & -5.6 & 848 & 370 & 53.5 & 10.3 & 539 & -3.3 & 352 & 276 & 71.6 & 53.0 \\
\hline 5 & “ & $\times \mathrm{R}-1001$ & 1036 & 4.6 & 848 & 580 & 45.1 & 22.2 & 452 & -18.9 & 352 & 321 & 34.3 & 28.4 \\
\hline 6 & AH8-A & $\times 2644-\mathrm{R}$ & 917 & -7.4 & 787 & 299 & 68.8 & 16.5 & 548 & -1.7 & 550 & 271 & 33.5 & -0.3 \\
\hline 7 & " & $\times 2284-\mathrm{R}$ & 1015 & 2.5 & 787 & 427 & 67.2 & 29.0 & 578 & 3.7 & 550 & 267 & 41.5 & 5.1 \\
\hline 8 & " & $\times 2280-R$ & 1026 & 3.6 & 787 & 317 & 85.8 & 30.4 & 559 & 0.3 & 550 & 239 & 41.9 & 1.8 \\
\hline 9 & “ & $\times 25711 R$ & 905 & -8.6 & 787 & 370 & 56.5 & 15.1 & 566 & 1.5 & 550 & 276 & 37.1 & 2.9 \\
\hline 10 & " & $\times \mathrm{R}-1001$ & 967 & -2.4 & 787 & 580 & 41.4 & 22.9 & 506 & -9.2 & 550 & 321 & 16.1 & -8.0 \\
\hline 11 & $2453-A$ & $\times 2644-R$ & 1134 & 14.5 & 285 & 299 & 288.3 & 278.9 & 642 & 15.2 & 547 & 271 & 57.1 & 17.5 \\
\hline 12 & " & $\times 2284-R$ & 1099 & 11.0 & 285 & 427 & 208.8 & 157.4 & 566 & 1.5 & 547 & 267 & 39.1 & 3.5 \\
\hline 13 & " & $\times 2280-R$ & 894 & -9.7 & 285 & 317 & 197.0 & 182.0 & 590 & 5.8 & 547 & 239 & 50.2 & 7.9 \\
\hline 14 & “ & $\times 25711 R$ & 875 & -11.7 & 285 & 370 & 167.0 & 136.3 & 474 & -15.0 & 547 & 276 & 15.3 & -13.3 \\
\hline 15 & " & $\times \mathrm{R}-1001$ & 1087 & 9.7 & 285 & 580 & 151.1 & 87.2 & 525 & -5.8 & 547 & 321 & 20.9 & -4.0 \\
\hline 16 & 0704-A & $\times 2644-R$ & 954 & -3.7 & 500 & 299 & 138.6 & 90.7 & 637 & 14.3 & 499 & 271 & 65.4 & 27.7 \\
\hline 17 & " & $\times 2284-R$ & 1113 & 12.4 & 500 & 427 & 140.1 & 122.6 & 562 & 0.8 & 499 & 267 & 46.9 & 12.8 \\
\hline 18 & " & $\times 2280-R$ & 1030 & 4.0 & 500 & 317 & 152.1 & 105.9 & 585 & 4.9 & 499 & 239 & 58.6 & 17.3 \\
\hline 19 & " & $\times 25711 \mathrm{R}$ & 1019 & 2.9 & 500 & 370 & 134.2 & 103.8 & 519 & -6.9 & 499 & 276 & 34.1 & 4.1 \\
\hline 20 & “ & $\times \mathrm{R}-1001$ & 1188 & 19.9 & 500 & 580 & 119.8 & 104.6 & 543 & -2.6 & 499 & 321 & 32.5 & 9.0 \\
\hline 21 & HА89A & $\times 2644-R$ & 1080 & 9.0 & 558 & 299 & 152.1 & 93.7 & 537 & -3.7 & 467 & 271 & 45.4 & 14.8 \\
\hline 22 & “ & $\times 2284-R$ & 957 & -3.4 & 558 & 427 & 94.4 & 71.6 & 611 & 9.6 & 467 & 267 & 66.3 & 30.6 \\
\hline 23 & " & $\times 2280-R$ & 899 & -9.2 & 558 & 317 & 105.6 & 61.2 & 477 & -14.4 & 467 & 239 & 35.2 & 2.1 \\
\hline 24 & " & $\times 25711 R$ & 818 & -17.4 & 558 & 370 & 76.3 & 46.7 & 435 & -22.0 & 467 & 276 & 17.0 & -7.0 \\
\hline 25 & " & $\times \mathrm{R}-1001$ & 1199 & 21.0 & 558 & 580 & 110.7 & 106.6 & 539 & -3.3 & 467 & 321 & 36.7 & 15.3 \\
\hline
\end{tabular}


Based on the calculated values for 2000, maximum values of hybrid vigor in the female lines were measured in the crosses of 2453-A (average 202\%), 0704-A (137\%), HA89-A (108\%), BAH-4 (71\%) and BAH-8 (64\%). In 2001, maximum heterobeltiosis values were found in the crosses of 2453-A (168\%), 0704-A (106\%), HA89-A (76\%) and BAH-4-A (51\%).

Among the restorer lines, the maximum regular heterosis in 2000 was determined in $2644-\mathrm{R}$ hybrids, $145 \%$ on average, followed by $2280-\mathrm{R}$ (123\%), 2284-R (116\%), 25711-R (98\%) and R-01001 (94\%). The maximum heterobeltiosis for oil yield was registered in $2644-\mathrm{R}$ hybrids (100\%), followed by $2284-\mathrm{R}$ (81\%), $2280-\mathrm{R}$ (80\%), R-01001 (69\%) and 25711-R (62\%). Based on the values for the two years, the crosses HA89-A $\times$ R-1001, 2453-A $\times 2644-\mathrm{R}, 0704-\mathrm{A} \times \mathrm{R}-1001$ and 0704-A $\times$ 2644-R, from the females 0704-A and 2453-A and the males R-1001 and 2644-R showed higher standard heterosis for oil yield than the other crosses.

The crosses had higher heterosis values for seed and oil yields in 2000 than in 2001. The main reason for the low yields in 2001 was a long drought that continued for a better part of the sunflower vegetation period. The heterosis values for the measured traits were higher in 2000, indicating that the climatic conditions in 2000 acted in a positive way, allowing a better expression of the potentials of the hybrids with regards to plant development. These highly heterotic crosses from 2000, involving highly significant $\times$ highly significant combiners, exhibited considerable additive genetic variance, which can be exploited for developing high yielding pure lines through progeny selection.

Negative heterotic values for hull rate are preferred by sunflower breeders. Maximum negative values of standard heterosis for hull rate, both in 2000 and in 2001 (-12.9\% and $-11.9 \%$, respectively), were registered in BAH8-A $\times 2644-\mathrm{R}$ followed by BAH8-A $\times 2280-\mathrm{R}(-12.5 \%$ in 2001) and BAH4-A $\times 2644-\mathrm{R}(-10.9 \%$ in 2001) (Table $3)$. Lower hull rate in the aforementioned crosses was accompanied by higher heterobeltiosis and regular heterosis values for this trait too.

Usefulness of crosses in heterosis breeding depends on their mean performance, SCA effects and magnitude of heterosis. Regarding the value of regular heterosis for hull rate, the most favorable performance among the females was shown by BAH-4 crosses followed by HA-89-A, 2453-A, BAH-8-A and 0704-A crosses. The crosses of 2644-R had the lowest heterotic values among the restorer lines, followed by the crosses of 2280-R 2284-R, 25711-R and R-01001.

Lowest heterobeltiosis values for hull rate were demonstrated by BAH-4-A crosses among the female lines, followed by HA-89-A, 2453-A, BAH-8-A and 0704-A crosses. The crosses of 2644-R exhibited highest negative heterotic values over better parent, followed by 2280-R, 2284-R, 25711-R and R-01001 crosses. BAH-4-A and BAH-8-A crosses had more favorable values of standard heterosis for hull rate than the other crosses in the research.

Heterosis values for oil content were low. The average standard heterosis ranged between $-6.2 \%$ and $9.4 \%$ in the experiment (Table 4 ). Low heterotic values 
were also observed for regular heterosis (between -10.5\% and 5.8\%) and heterobeltiosis (from $-12.8 \%$ to $3.0 \%$ ). Low heterosis results similar to these were obtained by Pathak et al. (1993) for sunflower oil content.

Table 3: Hull rate values of crosses in 2000 and 2001

\begin{tabular}{|c|c|c|c|c|c|c|c|c|c|c|c|c|c|c|}
\hline \multirow{2}{*}{\multicolumn{3}{|c|}{ CROSS $(A \times R)$}} & \multicolumn{6}{|c|}{2000} & \multicolumn{6}{|c|}{2001} \\
\hline & & & \multirow{2}{*}{$\begin{array}{c}\begin{array}{c}\text { HBT } \\
\%\end{array} \\
21.2\end{array}$} & \multirow{2}{*}{$\begin{array}{c}\begin{array}{c}\text { SHS } \\
\%\end{array} \\
-8.6\end{array}$} & \multirow{2}{*}{$\begin{array}{c}\begin{array}{c}\text { Fem } \\
\%\end{array} \\
19.1\end{array}$} & \multirow{2}{*}{$\begin{array}{c}\begin{array}{c}\text { Male } \\
\%\end{array} \\
21.2\end{array}$} & \multirow{2}{*}{$\begin{array}{l}\begin{array}{l}\text { HTS } \\
(\%)\end{array} \\
5.4\end{array}$} & \multirow{2}{*}{$\begin{array}{l}\begin{array}{c}\text { HTB } \\
(\%)\end{array} \\
0.2\end{array}$} & \multirow{2}{*}{$\begin{array}{c}\text { HBT } \\
\%\end{array}$} & \multirow{2}{*}{$\begin{array}{c}\begin{array}{c}\text { SHS } \\
\%\end{array} \\
-10.9\end{array}$} & \multirow{2}{*}{$\begin{array}{c}\begin{array}{c}\text { Fem } \\
\%\end{array} \\
25.9\end{array}$} & \multirow{2}{*}{$\begin{array}{c}\begin{array}{c}\text { Male } \\
\%\end{array} \\
23.9\end{array}$} & \multirow{2}{*}{$\begin{array}{c}\begin{array}{l}\text { HTS } \\
(\%)\end{array} \\
-19.3\end{array}$} & \multirow{2}{*}{$\begin{array}{c}\text { HTB } \\
(\%) \\
-22.4\end{array}$} \\
\hline 1 & BAH4-A & $\times 2644-R$ & & & & & & & & & & & & \\
\hline 2 & “ & $\times 2284-\mathrm{R}$ & 22.4 & -3.4 & 19.1 & 22.7 & 7.2 & -1.4 & 21.7 & -4.0 & 25.9 & 22.8 & -10.7 & -16.2 \\
\hline 3 & “ & $\times 2280-\mathrm{R}$ & 21.5 & -7.3 & 19.1 & 19.9 & 10.3 & 8.2 & 21.7 & -4.0 & 25.9 & 23.4 & -12.1 & -16.3 \\
\hline 4 & " & $\times 25711 R$ & 22.1 & -4.7 & 19.1 & 22.1 & 7.5 & 0.3 & 20.8 & -8.0 & 25.9 & 22.3 & -13.8 & -19.8 \\
\hline 5 & “ & $\times \mathrm{R}-1001$ & 24.1 & 3.9 & 19.1 & 22.6 & 15.7 & 6.8 & 23.1 & 2.2 & 25.9 & 23.7 & -6.9 & -10.9 \\
\hline 6 & BAH8-A & $\times 2644-R$ & 20.2 & -12.9 & 18.8 & 21.2 & 1.1 & -4.6 & 19.9 & -11.9 & 20.3 & 23.9 & -10.1 & -16.8 \\
\hline 7 & " & $\times 2284-\mathrm{R}$ & 22.8 & -1.7 & 18.8 & 22.7 & 9.8 & 0.4 & 21.8 & -3.5 & 20.3 & 22.8 & 1.0 & -4.3 \\
\hline 8 & “ & $\times 2280-R$ & 20.3 & -12.5 & 18.8 & 19.9 & 4.8 & 2.1 & 20.5 & -9.3 & 20.3 & 23.4 & -6.3 & -12.5 \\
\hline 9 & " & $\times 25711 R$ & 21.5 & -7.3 & 18.8 & 22.1 & 5.0 & -2.6 & 21.4 & -5.3 & 20.3 & 22.3 & 0.2 & -4.3 \\
\hline 10 & " & $\times \mathrm{R}-1001$ & 22.8 & -1.7 & 18.8 & 22.6 & 10.1 & 1.0 & 22.8 & 0.9 & 20.3 & 23.7 & 3.5 & -3.8 \\
\hline 11 & $2453-A$ & $\times 2644-\mathrm{R}$ & 24.0 & 3.4 & 24.9 & 21.2 & 4.2 & -3.6 & 22.1 & -2.2 & 21.1 & 23.9 & -1.9 & -7.6 \\
\hline 12 & “ & $\times 2284-R$ & 22.8 & -1.7 & 24.9 & 22.7 & -4.4 & -8.7 & 22.1 & -2.2 & 21.1 & 22.8 & 0.6 & -3.0 \\
\hline 13 & “ & $\times 2280-R$ & 22.9 & -1.3 & 24.9 & 19.9 & 2.4 & -8.0 & 23.2 & 2.7 & 21.1 & 23.4 & 4.1 & -1.0 \\
\hline 14 & “ & $\times 25711 \mathrm{R}$ & 23.2 & 0.0 & 24.9 & 22.1 & -1.1 & -6.8 & 23.3 & 3.1 & 21.1 & 22.3 & 7.2 & 4.3 \\
\hline 15 & " & $\times \mathrm{R}-1001$ & 23.5 & 1.3 & 24.9 & 22.6 & -1.1 & -5.8 & 21.9 & -3.1 & 21.1 & 23.7 & -2.2 & -7.4 \\
\hline 16 & 0704-A & $\times 2644-R$ & 22.0 & -5.2 & 23.3 & 21.2 & -1.1 & -5.5 & 23.0 & 1.8 & 24.8 & 23.9 & -5.5 & -7.1 \\
\hline 17 & “ & $\times 2284-R$ & 23.6 & 1.7 & 23.3 & 22.7 & 2.9 & 1.6 & 24.1 & 6.6 & 24.8 & 22.8 & 1.5 & -2.6 \\
\hline 18 & “ & $\times 2280-R$ & 23.0 & -0.9 & 23.3 & 19.9 & 6.7 & -1.1 & 24.2 & 7.1 & 24.8 & 23.4 & 0.2 & -2.5 \\
\hline 19 & “ & $\times 25711 \mathrm{R}$ & 24.7 & 6.5 & 23.3 & 22.1 & 9.1 & 6.2 & 24.2 & 7.1 & 24.8 & 22.3 & 2.9 & -2.2 \\
\hline 20 & “ & $\times \mathrm{R}-1001$ & 24.7 & 6.5 & 23.3 & 22.6 & 7.9 & 6.3 & 24.9 & 10.2 & 24.8 & 23.7 & 2.8 & 0.5 \\
\hline 21 & HA89A & $\times 2644-R$ & 21.9 & -5.6 & 20.9 & 21.2 & 4.3 & 3.4 & 22.2 & -1.8 & 24.3 & 23.9 & -7.7 & -8.5 \\
\hline 22 & “ & $\times 2284-R$ & 22.9 & -1.3 & 20.9 & 22.7 & 5.1 & 0.8 & 20.5 & -9.3 & 24.3 & 22.8 & -13.0 & -15.8 \\
\hline 23 & “ & $\times 2280-R$ & 22.4 & -3.4 & 20.9 & 19.9 & 10.2 & 7.6 & 21.6 & -4.4 & 24.3 & 23.4 & -9.6 & -11.3 \\
\hline 24 & “ & $\times 25711 \mathrm{R}$ & 22.2 & -4.3 & 20.9 & 22.1 & 3.3 & 0.5 & 22.7 & 0.4 & 24.3 & 22.3 & -2.8 & -6.7 \\
\hline 25 & " & $\times \mathrm{R}-1001$ & 23.2 & 0.0 & 20.9 & 22.6 & 6.8 & 2.8 & 23.7 & 4.9 & 24.3 & 23.7 & -1.2 & -2.5 \\
\hline
\end{tabular}

Standard average in 2000: $23.2 \%$; in 2001: $22.6 \%$

Heterotic values measured in the hybrids for oil content were mostly negative and there was no superiority of parents of crosses or standards. Highest values of regular heterosis was recorded by in the cross BAH4-A $\times 2644-\mathrm{R}$, of heterobeltiosis in the cross 0704-A $\times 2644-\mathrm{R}$ and of standard heterosis in the cross BAH8-A $\times$ 2644-R.

Based on average heterotic values of inbred lines, negative results were obtained in almost all of them for oil content. The ranking of the female lines was 
HA-89-A, BAH-4-A, 0704-A, 2453-A and BAH-8-A for regular heterosis and HA-89A, BAH-8-A, 0704-A, 2453-A and BAH-4-A for heterobeltiosis. The ranking of the restorer lines was 2644-R, 2280-R, 25711-R, 2284-R and R-01001 for regular heterosis and 2644-R, 2280-R, 25711-R, 2284-R and R-01001 for heterobeltiosis. Due to a drought that occurred in 2001 , low oil content values were measured in the sunflower crosses in the research.

Table 4: Oil contents of crosses in 2000 and 2001

\begin{tabular}{|c|c|c|c|c|c|c|c|c|c|c|c|c|c|c|}
\hline \multirow{2}{*}{\multicolumn{2}{|c|}{ CROSS $(\mathrm{A} \times$}} & \multirow{2}{*}{$\mathrm{R})$} & \multicolumn{6}{|c|}{2000} & \multicolumn{6}{|c|}{2001} \\
\hline & & & $\begin{array}{c}\text { HBT } \\
\%\end{array}$ & $\begin{array}{c}\mathrm{SHS} \\
\%\end{array}$ & $\begin{array}{c}\text { Fem } \\
\%\end{array}$ & $\begin{array}{c}\text { Male } \\
\%\end{array}$ & $\begin{array}{c}\text { HTS } \\
(\%)\end{array}$ & $\begin{array}{c}\text { HTB } \\
(\%)\end{array}$ & $\begin{array}{c}\mathrm{HBT} \\
\%\end{array}$ & $\begin{array}{c}\mathrm{SHS} \\
\%\end{array}$ & $\begin{array}{c}\text { Fem } \\
\%\end{array}$ & $\begin{array}{c}\text { Male } \\
\%\end{array}$ & $\begin{array}{c}\text { HTS } \\
(\%)\end{array}$ & $\begin{array}{c}\text { HTB } \\
(\%)\end{array}$ \\
\hline 1 & BAH4-A & $\times 2644-R$ & 48.8 & 0.9 & 49.4 & 49.2 & -1.1 & -1.3 & 44.6 & 5.1 & 38.7 & 42.2 & 5.8 & -2.2 \\
\hline 2 & " & $\times 2284-R$ & 47.8 & -1.2 & 49.4 & 50.1 & -3.9 & -4.6 & 42.6 & 0.2 & 38.7 & 43.3 & -1.7 & -11.2 \\
\hline 3 & " & $\times 2280-R$ & 47.9 & -1.0 & 49.4 & 52.6 & -6.0 & -8.9 & 43.3 & 1.9 & 38.7 & 41.2 & 5.0 & -1.1 \\
\hline 4 & " & $\times 25711 R$ & 45.4 & -6.2 & 49.4 & 45.0 & -4.0 & -8.2 & 43.3 & 1.9 & 38.7 & 42.2 & 2.5 & -5.4 \\
\hline 5 & " & $\times \mathrm{R}-1001$ & 45.5 & -6.0 & 49.4 & 52.3 & -10.5 & -13.0 & 40.9 & -3.8 & 38.7 & 41.9 & -2.4 & -9.4 \\
\hline 6 & BAH8-A & $\times 2644-\mathrm{R}$ & 47.9 & -1.0 & 50.5 & 49.2 & -4.0 & -5.2 & 46.5 & 9.4 & 44.8 & 45.2 & 2.8 & 1.9 \\
\hline 7 & “ & $\times 2284-\mathrm{R}$ & 46.6 & -3.7 & 50.5 & 50.1 & -7.4 & -7.7 & 43.3 & 1.9 & 44.8 & 46.4 & -6.7 & -9.7 \\
\hline 8 & " & $\times 2280-R$ & 49.0 & 1.2 & 50.5 & 52.6 & -5.0 & -6.9 & 45.3 & 6.6 & 44.8 & 44.3 & 2.1 & 0.9 \\
\hline 9 & “ & $\times 25711 \mathrm{R}$ & 45.9 & -5.2 & 50.5 & 45.0 & -3.9 & -9.1 & 43.2 & 1.6 & 44.8 & 45.3 & -4.6 & -5.6 \\
\hline 10 & “ & $\times \mathrm{R}-1001$ & 46.3 & -4.3 & 50.5 & 52.3 & -9.9 & -11.5 & 42.4 & -0.2 & 44.8 & 45.0 & -5.6 & -5.9 \\
\hline 11 & $2453-A$ & $\times 2644-\mathrm{R}$ & 47.4 & -2.1 & 42.7 & 49.2 & 3.1 & -3.8 & 42.5 & 0.0 & 44.1 & 44.9 & -5.2 & -6.8 \\
\hline 12 & " & $\times 2284-\mathrm{R}$ & 47.5 & -1.9 & 42.7 & 50.1 & 2.4 & -5.2 & 41.9 & -1.4 & 44.1 & 46.0 & -9.0 & -12.7 \\
\hline 13 & “ & $\times 2280-R$ & 48.7 & 0.6 & 42.7 & 52.6 & 2.3 & -7.4 & 41.5 & -2.4 & 44.1 & 43.9 & -5.4 & -5.8 \\
\hline 14 & " & $\times 25711 R$ & 45.6 & -5.8 & 42.7 & 45.0 & 4.1 & 1.3 & 40.5 & -4.7 & 44.1 & 44.9 & -9.8 & -11.5 \\
\hline 15 & " & $\times \mathrm{R}-1001$ & 48.0 & -0.8 & 42.7 & 52.3 & 1.1 & -8.2 & 43.4 & 2.1 & 44.1 & 44.6 & -2.6 & -3.7 \\
\hline 16 & 0704-A & $\times 2644-R$ & 47.0 & -2.9 & 44.6 & 49.2 & 0.1 & -4.6 & 43.9 & 3.3 & 41.8 & 43.7 & 0.3 & -3.9 \\
\hline 17 & “ & $\times 2284-\mathrm{R}$ & 47.0 & -2.9 & 44.6 & 50.1 & -0.7 & -6.3 & 42.8 & 0.7 & 41.8 & 44.9 & -4.6 & -10.8 \\
\hline 18 & “ & $\times 2280-\mathrm{R}$ & 48.6 & 0.4 & 44.6 & 52.6 & 0.0 & -7.6 & 43.3 & 1.9 & 41.8 & 42.8 & 1.2 & -1.1 \\
\hline 19 & " & $\times 25711 \mathrm{R}$ & 46.4 & -4.1 & 44.6 & 45.0 & 3.5 & 3.0 & 39.9 & -6.1 & 41.8 & 43.8 & -8.8 & -12.8 \\
\hline 20 & “ & $\times \mathrm{R}-1001$ & 47.5 & -1.9 & 44.6 & 52.3 & -1.9 & -9.2 & 41.1 & -3.3 & 41.8 & 43.4 & -5.4 & -8.9 \\
\hline 21 & HA89A & $\times 2644-R$ & 49.3 & 1.9 & 46.4 & 49.2 & 3.2 & 0.2 & 44.3 & 4.2 & 43.4 & 44.5 & -0.6 & -3.0 \\
\hline 22 & " & $\times 2284-R$ & 47.7 & -1.4 & 46.4 & 50.1 & -1.1 & -4.9 & 45.4 & 6.8 & 43.4 & 45.7 & -0.7 & -5.4 \\
\hline 23 & " & $\times 2280-\mathrm{R}$ & 48.9 & 1.0 & 46.4 & 52.6 & -1.1 & -7.0 & 43.9 & 3.3 & 43.4 & 43.6 & 0.7 & 0.3 \\
\hline 24 & " & $\times 25711 \mathrm{R}$ & 47.1 & -2.7 & 46.4 & 45.0 & 3.0 & 1.5 & 41.9 & -1.4 & 43.4 & 44.6 & -6.0 & -8.4 \\
\hline 25 & “ & $\times \mathrm{R}-1001$ & 49.0 & 1.2 & 46.4 & 52.3 & -0.7 & -6.4 & 42.5 & 0.0 & 43.4 & 44.3 & -4.0 & -5.8 \\
\hline
\end{tabular}

\section{CONCLUSION}

The range of heterosis was quite considerable, indicating a high vigor potential of the crosses in the research. Most of the crosses exhibited high heterosis espe- 
cially for seed and oil yields. However, mean heterosis was comparatively low for hull and oil contents. The most favorable performance was shown for seed yield (288.3\%).

The study on heterosis in sunflower showed that the crosses with favorable characteristics such as oil and seed yields, oil and hull contents could be bred from correctly selected parents. The cross $2453-\mathrm{A} \times 2644-\mathrm{R}$ reached the breeding aim mentioned above, especially for high vigor in seed and oil yields. The following crosses demonstrated high hybrid vigor and superior performance over standard hybrids in the studied characteristics: the cross $2453-\mathrm{A} \times 2284-\mathrm{R}$ for seed and oil yields, the crosses BAH4-A $\times 2644-\mathrm{R}$ and $\mathrm{BAH}-8-\mathrm{A} \times 2644-\mathrm{R}$ for thin hull rate and high oil content.

The evaluation of inbred lines based on all three criteria of heterosis showed that the crosses of the female line 2453-A and the male line 2644-R revealed higher hybrid vigory than the other lines regarding the characteristics examined in this research. The male line 2280-R with regard to all measured traits, the female line 704-A with regard to seed and oil yields and the female lines HA-89-A and BAH-4-A with regard to high oil content and low hull rate could be used for increasing hybrid vigor in future sunflower breeding programs.

\section{REFERENCES}

Fick, G.N. and Miller, J.F., 1997. Sunflower breeding. In: A.A. Schneiter (ed.) Sunflower Technology and Production. ASA, SCSA, and SSSA Monograph. No: 35. Madison, WI, 395-440.

Giriraj, K. and Virupakshappa, K., 1992. Heterotic effect for seed yield and component characters in sunflower over seasons. In: Proceedings of the $13^{\text {th }}$ Int. Sunflower Conf. Pisa, Italy, September 7-11, 1043-1047.

Guo-Zhan, L. and Chun-Fang, G., 1985. Heterosis and its utilization in sunflower. In: Proc. $11^{\text {th }}$ Int. Sunflower Conf. Mar Del Plata, Argentina, March 10-13, 805.

Kaya, Y., 2004. Sunflower Breeding, Seed Industry and Future Directions in Turkey. Proc. of $16^{\text {th }}$ Int. Sunflower Conf. August 29-Sept. 2, Fargo, USA, 465-72.

Miller, J.F., 1987. Sunflower. In: W.R. Fehr (ed.) Principles of Cultivar Development. Vol. II. Macmillan Publ. Co. New York, USA, 626-668.

Miller, J.F. and Fick, G.N., 1997. Sunflower genetics. In: A.A. Schneiter (ed.) Sunflower Technology and Production. Agron. Monogr. 35, ASA, CSSA and SSSA, Madison, WI, USA, 441-495.

Pathak, A.R., Singh, B. and Kukadia, M.U., 1983. Heterosis in sunflower. Gujarat Agric. Univ. Res. J. 9(1): 62-65.

Reddy, P.S., Reddy, M.V., Lawrence, M. and Sarma, N.D.R.K., 1985. Heterobeltiosis for seed yield and oil content in sunflower (H. annuus L.). Indian J. Genet. \& Plant Breeding 45( 1): 166-170.

Singh, S.B., Labana, K.S. and Virk, D.S., 1984. Heterosis in variety $\times$ inbred crosses of sunflower. Crop Improvement 11(1): 33-38.

Zhao-Cheng, X., Duo, L., Gui-Zhi, W. and Jie, Q., 1988. Applied theory of relative heritability to calculate the heterosis of sunflower. In Proc. of the $12^{\text {th }}$ Int. Sunflower Conf. Novi Sad, Yugoslavia, July 25-29, 484-87.

Virupakshappa, K., Nehru, S.D., Gowda, J. and Hedge, S., 1997. Selection of testers for combining ability analysis and relationship between per se performance and GCA in sunflower (H. annuus L.). Helia 20(26): 79-88. 


\title{
POTENCIA HÍBRIDA DE GIRASOL (Helianthus annuus L.)
}

\author{
RESUMEN
}

Los rendimientos de semilla y aceite incrementados son la meta principal de la mayoría de los programas de selección de girasol. La utilización de heterosis es la meta principal en la selección de girasol. Los objetivos de esta investigación eran de determinar el rendimiento de las variedades de girasol y de medir la potencia híbrida en los híbridos. Los híbridos de girasol fueron evaluados sobre la base de cuatro componentes de rendimiento importantes, rendimiento de semilla, heterosis estándar y regular y heterobeltiosis. Las investigaciones fueron realizadas en los años 2000 y 2001 en las condiciones de la localidad de Edirne, Turquía. Sobre la base de las observaciones hechas, el rendimiento de la semilla de los híbridos cambió de 37 a $245 \mathrm{~kg} \mathrm{ha}^{-1}$; el rendimiento de aceite de 17,6 a $118,8 \mathrm{~kg} \mathrm{ha}^{-1}$; el contenido de aceite de 38,0 a $50,8 \%$; el contenido de vaina de 19,2 a $27,1 \%$. Los valores más altos de heterosis $(288,3 \%)$ y de heterobeltiosis $(98 \%)$, fueron obtenidos para el rendimiento de aceite y de heterosis estándar (21.2\%) para el rendimiento de la semilla. Los valores más bajos de heterosis $(-19.3 \%)$ y de heterobeltiosis $(-22.4 \%)$ fueron obtenidos para el contenido de vaina, y el más bajo heterosis estándar (-22.0\%) fue obtenido para el rendimiento de aceite. El mejor rendimiento de las propiedades estudiadas, demostró la undécima combinación (2453-A × 2644-R). Entra las líneas consanguíneas investigadas, los híbridos con la línea 2453-A como madre, y línea 2644-R como padre, tenían incrementada potencia híbrida en relación con los demás híbridos.

VIGUEUR HYBRIDE DU TOURNESOL (Helianthus annuus L.) RÉSUMÉ

Augmenter les rendements d'huile et de graines est la priorité de la plupart des programmes de culture du tournesol. Utiliser l'hétérosis est le but principal de la culture du tournesol. Les buts de cette recherche étaient de déterminer les performances des sortes de tournesol et de mesurer la vigueur hybride. Les hybrides de tournesol ont été évalués d'après quatre composants importants, le rendement, le rendement en graines, l'hétérosis standard et régulier et l'hétérobeltiosis en 2000 et 2001 dans les conditions de la localité d'Edirne, Turquie. Cette recherche a permis d'observer que le rendement en graines des hybrides avait changé de 37 à $245 \mathrm{~kg} \mathrm{ha}^{-1}$; le rendement en huile de 17,6 à $118,8 \mathrm{~kg} \mathrm{ha}^{-1}$; le contenu d'huile de 38,0 à $50,8 \%$; le contenu des écailles de 19,2 à $27,1 \%$. Les plus grandes valeurs d'hétérosis $(288,3 \%)$ et d'hétérobeltiosis (98\%) ont été obtenues pour le rendement d'huile et celles d'hétérosis standard pour le rendement en graines. Les valeurs d'hétérosis les plus faibles $(-19,3 \%)$ et d'hétérobeltiosis $(-22,4 \%)$ ont été obtenues pour le contenu des écailles et l'hétérosis standard le plus faible $(-22,0 \%)$ pour le rendement d'huile. La onzième combinaison $(2453-\mathrm{A} \times 2644-\mathrm{R})$ a présenté la meilleure performance des caractéristiques observées. Parmi les souches pures examinées, les hybrides de la souche mère 2453-A et de la souche père 2644-R ont présenté une vigueur hybride supérieure à celle des autres hybrides. 